\title{
Model-driven Approach to the Optimal Configuration of Time-triggered Flows in a TTEthernet Network
}

\author{
Sofiene Beji ${ }^{1}$, Abdelouahed Gherbi ${ }^{2}$, John Mullins $^{1}$, and Pierre-Emmanuel \\ Hladik $^{3}$ \\ 1 Department of Computer and Software Engineering \\ École Polytechnique de Montréal \\ firstname.lastname@polymtl.ca \\ 2 Department of Software and IT Engineering, École de Technologie Supérieure, \\ Montréal, QC, Canada \\ abdelouahed.gherbi@etsmtl.ca \\ 3 LAAS-CNRS, Université de Toulouse, CNRS, INSA, Toulouse, France \\ pehladik@laas.fr
}

\begin{abstract}
The SAE standard Time-triggered Ethernet defines a strong networking infrastructure, which supports the engineering of avionic systems. Avionic functions are often designed independently and integrated to form the avionic system. The iterative integration approach helps in controlling the design complexity of evolving avionic systems and aims at minimizing the cost associate with the reconfiguration of scheduling parameters of already integrated parts. On the other hand, the iterative approach requires to specify and manage a huge set of constraints, which are then solved to compute the optimal scheduling parameters. In this paper, we focus on this issue of manual specification of these constraints by the system engineer. We propose a model-driven approach, which provides the required abstractions and automation to support the system engineer in using effectively the iterative integration approach. The abstractions consist in a metamodel, which describes the system at a given integration step and a metamodel for the constraints. The automation consists in a model transformation which enables generating automatically the relevant constraints at integration step.
\end{abstract}

Keywords: Time-Triggered Ethernet, IMA, Model-driven approach, Metamodel, Model transformation

\section{Introduction}

Avionic embedded systems are now engineered following the principles of Integrated Modular Avionics (IMA) architecture [1]. The IMA architecture is characterized essentially by the sharing of distributed computing resources called modules. Since avionic systems are inherently safety-critical systems, sharing these resources requires to guarantee some safety properties such as the collision-free. 
Moreover, IMA-based avionic systems are distributed systems which depend on a robust and deterministic networking infrastructure. The Avionic Full Duplex Switched Ethernet (AFDX) [2] has long been adopted as a networking standard for the avionic systems. Therefore, the IMA and AFDX became the main components of a typical architecture model for the recent civil aircrafts such as $B 787$ and $A 380$. More recently, the SAE standard Time-Triggered Ethernet (TTEthernet) is emerging as a new standard of the avionic network [3]. With respect to AFDX, the TTEthernet standard enables to achieve a best usage of the network resources and is more deterministic. In particular, the TTEthernet network schedule is established off-line.

The avionic functions designed independently need to be integrated within an existing system deployed on a TTEthernet-networked IMA architecture. The integration of avionic system is a complex engineering task. We have presented in [4] an iterative integration approach, which enables the integration of multiple IMA partitions as well as TTEhernet frames. This approach addresses the issue of finding an appropriate scheduling for the partitions and frames. The synthesized schedule may require the reconfiguration of the already integrated frames or partitions which lead to a supplementary re-certification cost that we aim to minimize. We consider this issue as Constraints Optimization Problem (COP) [13] where we satisfy not only a set of constraints but also we optimize the reconfiguration cost function.

In order for avionic engineers to use the iterative integration approach, they have to specify both the existing system and the new avionic functionality using a set of formal constraints. These constraints can then solved effectively using Constraints Programming (CP) techniques. The number and complexity of these constraints grow up very sharply even for small system examples. Therefore, using effectively the iterative integration approach faces a challenging constraints management complexity. In order to overcome this issue, we propose in this paper a model-driven engineering approach, which provides the required abstractions (i.e. metamodels) and defines the transformation process to enable the automatic generation of these constraints.

The paper is organized as follows. Section 2 presents the background knowledge and the model for the iterative integration problem. In Section 3, we introduce our model-driven engineering approach. We dedicate Section 4 and Section 5 to present the meta-models of our approach. We define in Section 6 the transformation process that generates the constraint program for a given integration problem. Our case study is presented in Section 7. We present the related works in Section 8 and we conclude the paper in Section 9.

\section{Iterative Integration on TTEthernet Networks: Backgrounds and Model}

The TTEthernet is a layer 2 protocol standardized under SAE AS6802 [3]. It defines a strategy of clock synchronization in a distributed system. The TTEthernet supports two classes of traffic: time-triggered traffic and event-triggered 
traffic. The time-triggered traffic is relevant mainly for the critical applications. In this work, we are only interested in time-triggered (TT) traffic. In contrast to the event-triggered traffic, the time-triggered one is static and fixed time windows are reserved for the transmission of each frame on a given dataflow link.

A TTEthernet network can be represented by a graph $\mathcal{G}=(\mathcal{V}, \mathcal{E})$ where $\mathcal{V}$ represents the nodes of the network and $\mathcal{E}$ the set of physical links. The nodes are of two types: the set of End-Systems $(E S)$ and the set of Network-Systems $(N S)$ Each physical link connecting two nodes defines a bidirectional communications, each of which is a dataflow link.

A TTEthernet frame $f_{i}$ is communicated from its source to its destinations throughout fixed paths called virtual links. A virtual link $v l_{i}$ is therefore associated with each frame $f_{i}$ and defines a tree structure where its nodes are a set of dataflow links. The root element of this tree is the first link on which a frame $f_{i}$ is transmitted, denoted $\operatorname{first}\left(f_{i}\right)$. The leaves are the last dataflow links on which the frame $f_{i}$ is transmitted, designated $\operatorname{last}\left(f_{i}\right)$. We denote by next $\left(f_{i}, l\right)$ the next dataflow links on which the frame $f_{i}$ is transmitted taking as reference the dataflow link $l$. We denote by $f_{i}^{l}$ the transmission of the frame $f_{i}$ on the dataflow link $l, f_{i, k}^{l}$ the $k^{\text {th }}$ instance of the frame $f_{i}$ on the dataflow link $l$ and by $\mathcal{L}_{i}$ the set of dataflow links on which $f_{i}$ is defined.

The transmission of each frame $f_{i}$ is characterized by the parameters: the period of $f_{i}, f_{i}$.Period, and its transmission delay on a dataflow link $f_{i}$. Length. The periodic pattern describing the communication of all the frames is called hyper-period $(H P)$ and defined as the least common multiple of all frame periods. We denote by Instances $\left(f_{i}\right)$ the number of instances considered for the frame $f_{i}$ and formally defined as $\frac{H P}{f_{i} \text { Period }}$. The schedule of the $k^{\text {th }}$ instance of the frame $f_{i}$ on the dataflow link $l$ is determined by the variable $f_{i}^{l}$. Offset which designate the offset time with the respect to the beginning of $H P$. The offsets are the only variables of the integration problem.

In the iterative integration problem as defined in [9] and [4], we have some configured applications which communicate through a TTEthernet network and we want to integrate new ones. To ensure the real-time requirements, we may reconfigure the scheduling of the previous ones. We focus in the scope of this paper on the reconfiguration of the network. This reconfiguration induces an additional cost of the re-certification of the system. We designate by $\operatorname{Cost}\left(f_{i}\right)$ the cost of reconfiguring a frame $f_{i}$ on a given dataflow link. We denote by $\mathcal{F}$ the set of considered frames, $\mathcal{F}_{\text {old }}$ the set of configured frames and $\mathcal{F}_{\text {new }}$ the set of frames to configure.

For a configured frame $f_{i}$, we denote by $f_{i / b}^{l}$. Offset $(k)$ the offset of the $k^{\text {th }}$ instance of the frame $f_{i}$ on the dataflow link $l$ before the integration and by $f_{i / a}^{l}$. Offset $(k)$ this offset after the integration. When it is clear from the context, we simply designate the offset after the integration by $f_{i}^{l}$. Offset $(k)$. We define by $R_{i}^{l}(k)$ the reconfiguration function that returns 1 if the $k^{t h}$ instance of frame $f_{i}$ on the dataflow link $l$ is reconfigured and 0 otherwise. The goal of the iterative integration problem is then to minimize the total reconfiguration cost of the 
network. The reconfiguration cost of a frame instance $f_{i, k}^{l}$ is equal to $\operatorname{Cost}\left(f_{i}\right) \times$ $R_{i}^{l}(k)$

\section{Overall Approach}

We present in this section our model-driven engineering approach to automatically generate the constraints program that solves the problem of a given integration step. As shown by Figure 1, our approach relies on the definition of two meta-models. The first one, called the Integration Specification Meta-Model, characterizes an iterative integration problem on TTEthernet networks. The second one called CP Integration Meta-Model defines the CP formalization to solve this problem. An instance of the first meta-model describes a real case of the iterative integration problem. We specify in this instance the configured frames and the frames to be configured and how they are deployed on the network architecture. An instance of the second meta-model models the CP program that solves a specific iterative integration problem. A transformation tool, which relies on the meta-models, enables transforming a given instance of the Integration Specification meta-model to the corresponding CP model. The latter is then transformed to a $\mathrm{CP}$ code structured following the targeted $\mathrm{CP}$ language specification and solved by a CP solver to find a new optimal configuration. By defining an intermediate $\mathrm{CP}$ model before generating the $\mathrm{CP}$ integration code, our approach can target different CP solvers. To test our approach, we have used MiniZinc [6] as target CP language. We specify in the following section the Integration Specification meta-model.

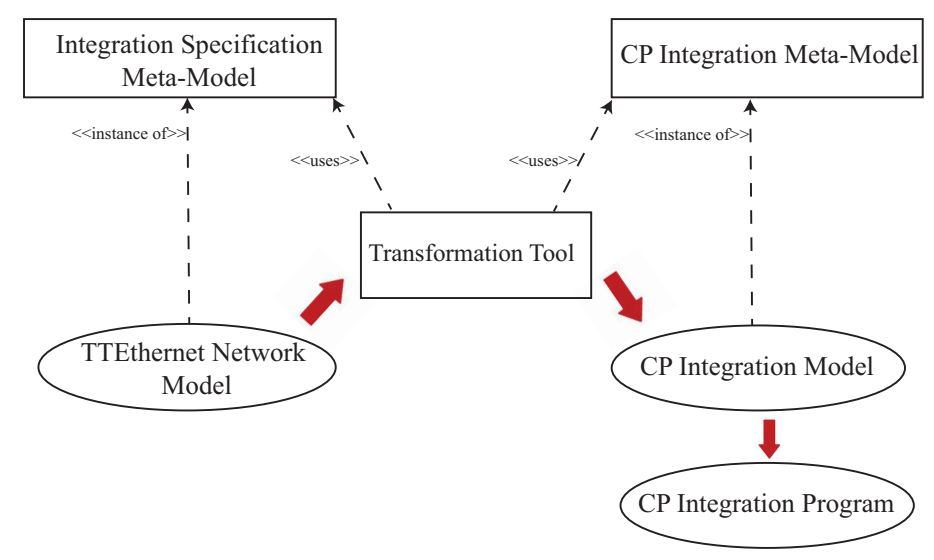

Fig. 1: Approach Overview 


\section{Integration Specification Meta-Model}

This meta-model is depicted in Figure 2. An integration problem is based on the definition of integration steps, which are represented by the metaclass IntegrationStep. This metaclass has an attribute step which indicates the step order of the integration. It has also the attributes switchTreatmentDelay and $H P$ which designate respectively the required delay for a switch to handle a frame and the Hyper-Period for all the frame periods. An IntegrationStep is composed of a set of frames to configure and the set of already configured frames if any. A ConfiguredFrame differs from a FrameToConfigure by the indication of the actualOffsets attribute which indicates the schedule before the integration of each instance of the considered frame on each dataflow link. A Frame is characterized by several attributes including period, length, reconfigurationCost, nbInstances, etc. A

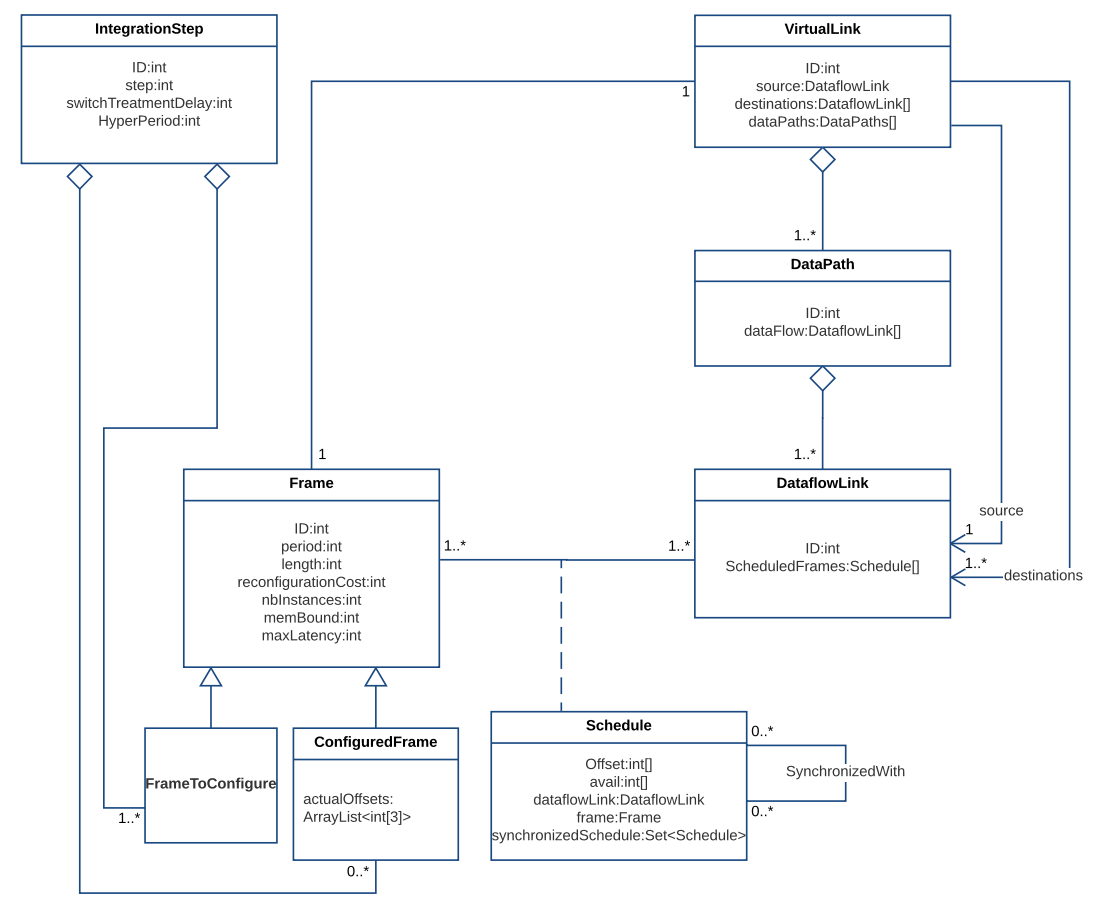

Fig. 2: The Integration Specification Meta-Model

VirtualLink is associated with each frame. Each VirtualLink is characterized by a source and destinations DataflowLink. A VirtualLink can be hence composed of a number of DataPaths. Each DataPath defines a path from the source to one destination. It is constituted by the adjacent sequence of a dataflow links. A Schedule characterizes the allocation of time windows of each frame on each 
dataflow link. Obviously, many frames can be scheduled on a dataflow link and a frame is transmitted on the different dataflow links that defines its associated virtual link. The Schedule of a frame on a dataflow link may be synchronized with another of the same one on another dataflow link. This case occurs when a frame must be relayed simultaneously on different dataflow links

\section{CP Integration Meta-Model}

Our CP Integration Meta-Model is depicted by Figure 3. It is composed of a set of VariableDeclaration metaclasses, a set of Constraint metaclasses and a SolveItem metaclass. For readability purpose, we do not include in Figure 3 the comprehensive set of the relationships between these metaclasses. A SolveItem models a directive to the solver, which consists in the definition of two attributes, type, which is the type of the problem either a satisfaction or minimization problem; and objective, which is the optimized objective in the case of an optimization problem. In our case, the optimized objective is the reconfiguration cost.

\subsection{Variables Declaration}

As shown by Figure 3, three types of variables are considered to solve the integration problem. The metaclass FrameInstanceOffsets specifies the offsets of the different frame instances of a frame on a dataflow link. FrameInstanceOffsets has the two attributes name and type.The attribute name is of type FrameInstanceName and defines the name of the frame instances on one dataflow link. The name is therefore uniquely identified by the IDFrame and the IDLink. The attribute type is of type int []. The metaclass FrameInstanceReconfig captures the information whether the offsets of the already configured frame instances are changed after the integration. It has two attributes name which is also the name of the considered frame instances on a dataflow link and the attribute type of type bool[]. The metaclass ReconfigurationCost specifies the cost of the schedule after the integration.

\subsection{Constraints}

To solve the integration problem, we consider nine types of constraints illustrated in Figure 3. We introduce for the definition of these constraints a new type Quantifier which quantifies the instance order of a frame and has four attributes: (1) ident which gives an identification name for the quantifier, (2) type which specifies the type of the quantifier (e.g. exists or forall), (3) min the minimum value of the quantifier and (4) $\max$ the max value of the quantifier.

Contention-Free Constraints: A Contention-Free Constraint expresses the mutual exclusion of a transmission on a dataflow link. Given two frames $f_{i}$ and $f_{j}$ transmitted on a dataflow $\operatorname{link} l$, the end of transmission of an instance of $f_{i}$ 


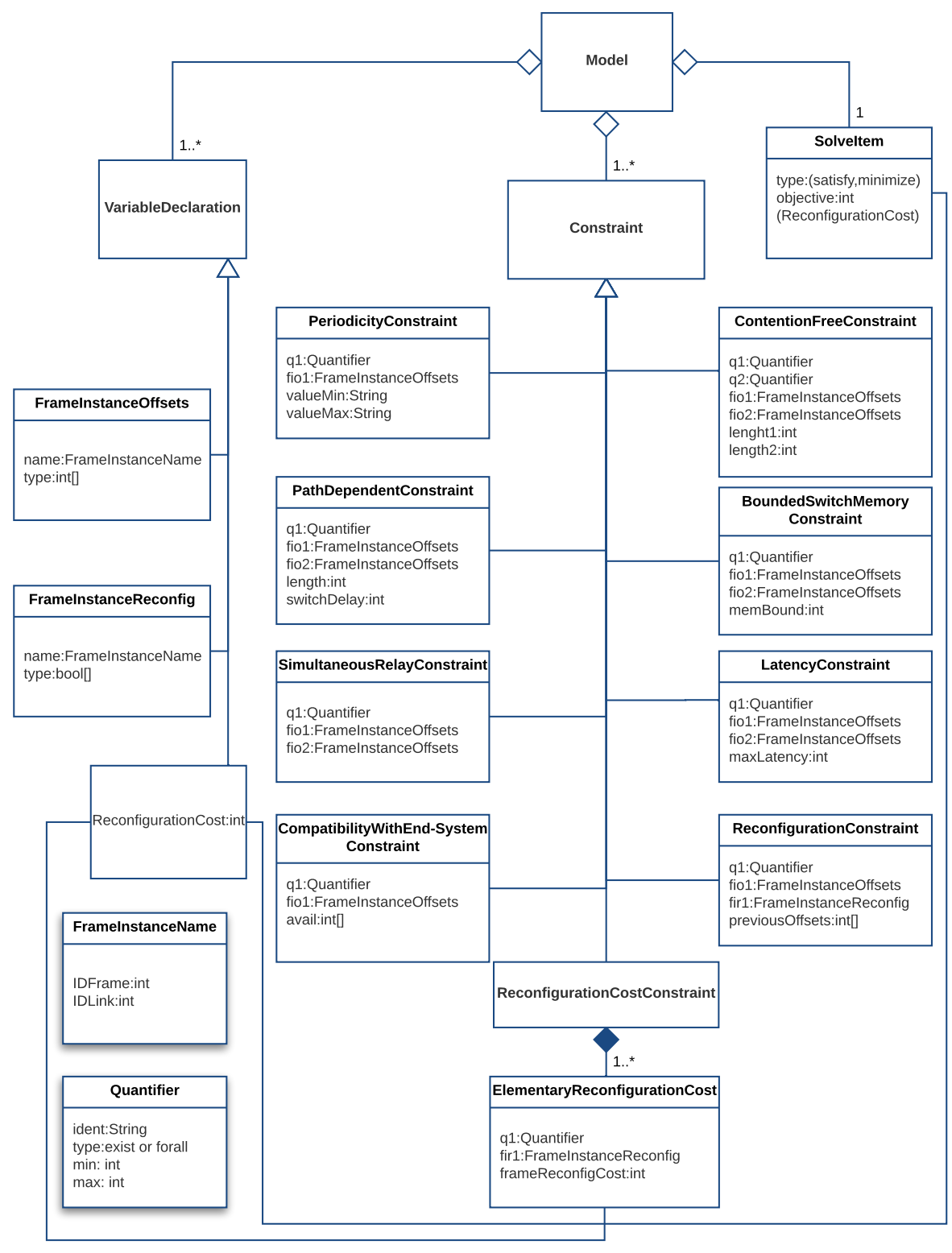

Fig. 3: The CP Integration Meta-Model

on $l$ occurs before the beginning of transmission of a given frame instance of $f_{j}$ 
or vice versa. The contention free constraints can be formalized as follows:

$$
\begin{aligned}
& \forall f_{i}, f_{j} \in \mathcal{F}, \forall l \in \mathcal{L}_{i} \cap \mathcal{L}_{j}, \forall k \in\left[1 . \text { Instances }\left(f_{i}\right)\right], \forall k^{\prime} \in\left[1 . . \text { Instances }\left(f_{j}\right)\right], \\
& \left(f_{i}^{l} . \text { Offset }(k)+f_{i} . \text { Length } \leq f_{j}^{l} \text {.Offset }\left(k^{\prime}\right)\right) \vee \\
& \left(f_{j}^{l} . \text { Offset }\left(k^{\prime}\right)+f_{j} . \text { Length } \leq f_{i}^{l} \text {.Offset }(k)\right)
\end{aligned}
$$

In the CP Integration Meta-Model, a Contention-Free Constraint is modeled by the class ContentionFreeConstraint which has the attributes (1) $q 1$ and $q 2$ as two Quantifiers on respectively the instance order $k$ and $k^{\prime}$, (2) fio1 and fio. the offsets of respectively $f_{i}^{l}$ and $f_{j}^{l}$ and (3) length1 and length2 to designate the transmission delays of $f_{i}$ and $f_{j}$ on a dataflow link.

Path-Dependent Constraints: We introduce this constraint to express the sequential transmission of a frame $f_{i}$ along a data path of the virtual link $v l_{i}$. Formally this constraint is defined as follows:

$$
\begin{aligned}
& \forall f_{i} \in \mathcal{F}, \forall l^{\prime} \in \operatorname{next}\left(f_{i}, l\right), \forall k \in\left[1 . \text { Instances }\left(f_{i}\right)\right], \\
& \left(f_{i}^{l} \text {.Offset }(k)+f_{i} . \text { Length }+ \text { switch_delay }\right) \leq\left(f_{i}^{l^{\prime}} \text {.Offset }(k)\right)
\end{aligned}
$$

where, switch_delay denotes the processing delay of a frame by a switch. A PathDependent constraint is specified in our meta-model by the metaclass PathDependentConstraint, which has the following attributes: (1) $q 1$ as a Quantifier on the instance order $k$ of $f_{i},(2)$ fio1 and fio 2 the offsets of respectively $f_{i}^{l}$ and $f_{i}^{l^{\prime}}$, (3) length the parameter $f_{i}$.Length and (4) switchDelay the processing delay of a frame by a switch.

Latency Constraints In order to ensure that frames meet their deadline requirements, we define latency constraints. These constraints bound the transmission delay of a frame along their datapaths and are formalized as follows

$$
\begin{aligned}
& \forall f_{i} \in \mathcal{F}, \forall l \in \operatorname{last}\left(f_{i}\right), \forall k \in\left[1 . . \text { Instances }\left(f_{i}\right)\right], \\
& f_{i}^{l} . \text { Offset }(k)-f_{i}^{\text {first }\left(f_{i}\right)} . \text { Offset }(k) \leq \text { max_latency }_{i}
\end{aligned}
$$

We represent a latency constraint in our CP meta-model by the metaclass LatencyConstraint which has the attributes (1) $q 1$ to represent the instance order $k$, (2) fio1 to designate the offsets of $f_{i}^{f i r s t\left(f_{i}\right)}$, (3) fio? to designate the offsets of $f_{i}^{l}$ and (4) maxLatency the maximal tolerated bound of latency.

Reconfiguration Constraint The reconfiguration constraints detect if the already configured frames are reconfigured after the integration of new frames. The reconfiguration constraints are formalized as follows

$$
\begin{aligned}
& \forall f_{i} \in \mathcal{F}_{\text {old }}, \forall l \in \mathcal{L}_{i}, \forall k \in\left[1 . . \text { Instances }\left(f_{i}\right)\right], \\
& \left(f_{i / b}^{l} . \text { Offset }(k)=f_{i / a}^{l} . \text { Offset }(k)\right) \Leftrightarrow\left(R_{i}^{l}(k)=0\right)
\end{aligned}
$$


In the CP Meta-Model, a reconfiguration constraint is modeled by the metaclass ReconfigurationConstraint which has the attributes (1) $q 1$ to represent the instance order $k$, (2) fio1 to designate the offsets after the integration (3) fir1 to designate the reconfiguration variables $R_{i}^{l}(k)$ and (4) previousOffsets which contains the previous offsets of $f_{i}$ on the dataflow link $l$.

\section{Model Transformation Process}

We detail in this section the transformation rules implemented in our Transformation Tool to generate automatically the CP Integration Model. We note by ModelIn the integration specification model and by ModelOut the CP integration model. In the remainder, we detail the transformation rule corresponding to each component of a CP Integration model.

\subsection{Variables}

For the variables of the $\mathrm{CP}$ Model, we define the transformation rule given by Algorithm 1 that generates the FrameInstanceOffset instances. In Line 1, we select from the input model an instance of the metaclass Schedule, denoted by $A$. We create in Line 2 an instance of the FrameInstanceOffsets metaclass corresponding to $A$ that we denote by $B$. In Line $3-5$, we assign the relevant attributes.

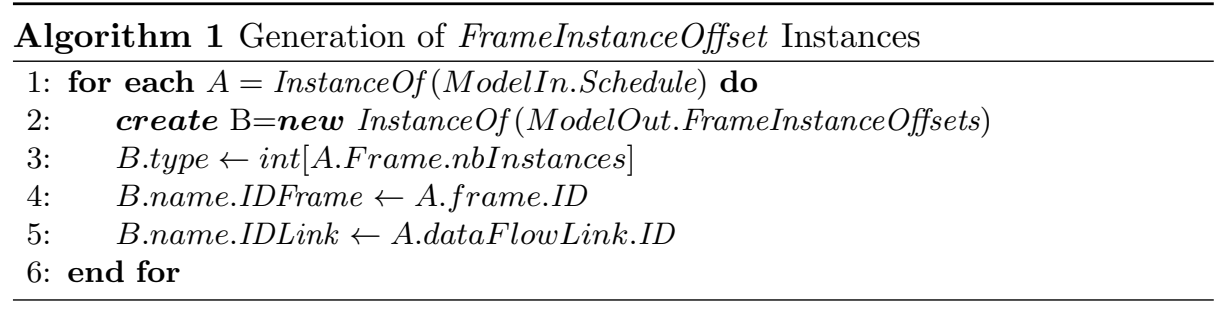

\subsection{Constraints}

For the constraints, we present only the transformation rule that define the ContentionFreeConstraint instances. This rule is specified by Algorithm 2. In Line 1-2, we select from the input model two instances $A$ and $B$ of the metaclass Schedule. In order to define correctly a Contention-Free Constraint, we must check in Line $3-4$ that $A$ and $B$ are two instances that define a schedule of two different frames in the same dataflow link. To ensure constraint unicity, we impose that the $I D$ of the frame associated with $A$ is inferior that of $B$. We create then in Line 4 a new instance $C$ of the ContentionFreeConstraint metaclass. In Line $5-6$, we define the two quantifiers of the created instance C. $q 1$ is reserved 
for the schedule of the frame associated with the instance $A$ and $q 2$ for that of B. In Line $7-11$, we define the attributes fio1 and length1 that correspond respectively the schedule and the transmission delay of the frame associated with $A$. Similarly, in Line $12-16$, we define the schedule and the transmission delay of the frame associated with $B$.

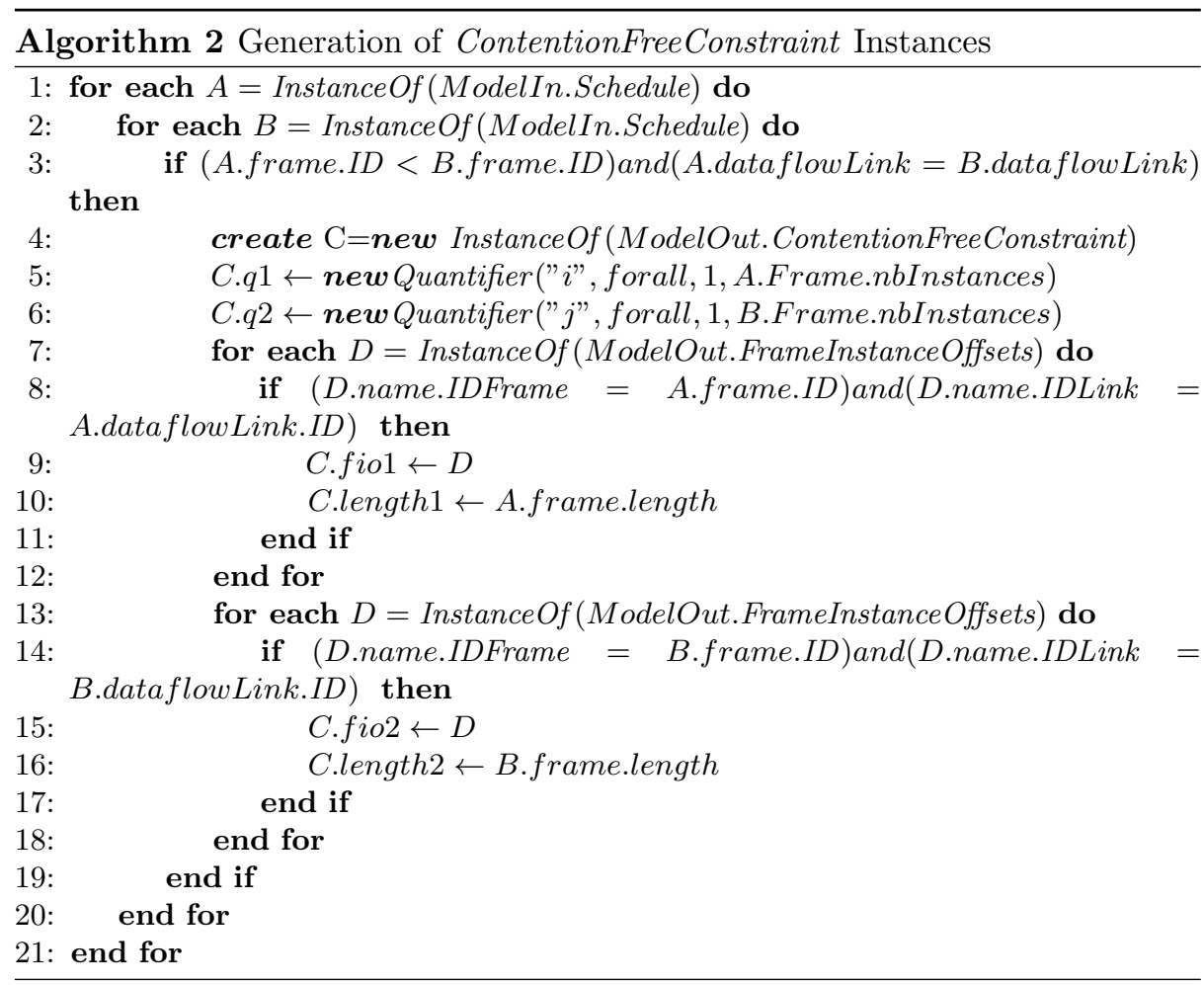

\subsection{SolveItem}

For the SolveItem, we have one instance by a CP Model (i.e. a singleton). We generate this instance by following the Algorithm 3. In Line 1, we check the existence of any already configured frame. This allows the definition of the nature of the problem. If no frame is already configured which is the case in Line $2-3$, we assign the value satisfy to the type of the problem. In the contrary case, shown in Line $5-6$, the problem is rather of type minimize and the objective to minimize is the Reconfiguration Cost. 


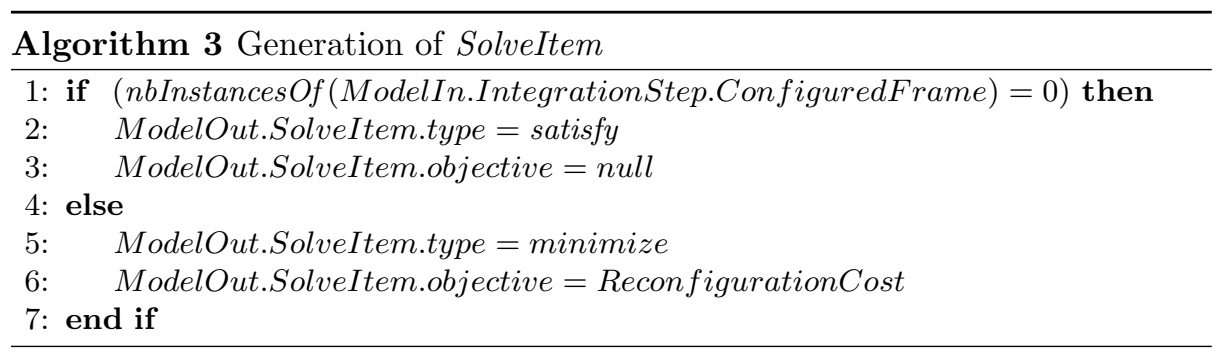

\section{Case Study}

In this section, we illustrate our model-driven engineering approach through the integration of the communication part of the distributed system whose physical architecture is illustrated by Figure 4. We identify in this figure the different dataflow links by numbers and we illustrate the direction of each flow by a dashed arrow.

The temporal characterization of the frames is given by Table 1 . We propose as shown by the first column to integrate the set of frames in three integration steps. The IDs of the frames are indicated in the second column. The frame periods are given by the third column. We reserve the fourth column to the indication of the information availability dates at the ES level. The fifth column indicates the transmission delays of the frames on a dataflow link. We indicate in the last column the structure of the virtual link. We adopt in this field the notation $l_{s}-\left\{l_{d_{1}}, \ldots, l_{d_{n}}\right\}$ to indicate that the associated frame is transmitted first in $l_{s}$ and the simultaneously in $l_{d_{1}}$ to $l_{d_{n}}$. The reconfiguration cost of each frame in this example is equal to 1.

In the following, we use this case study to illustrate through two examples: (1) the integration specification in input as instance of the Integration Specification Meta-Model, (2) an instance of the CP Integration Meta-Model corresponding to the spec input, and (3) the associated MiniZinc code relative the to the CP model.

Although this example illustrates the integration of only 26 virtual links, we note that the resolution of each integration step requires about one thousand of code lines. We only illustrate some relevant aspects of our approach using two small examples. We set in the first example as goal to show the generation of some CP variables and frame constraints through the example of a ContentionFree Constraint. In the second example, we explain a constraint that exploits the structure of the virtual link.

\subsection{Example 1}

Focusing in the first integration step and more precisely the integration of frames $f_{3}$ and $f_{4}$, we notice that $f_{3}$ is scheduled on the dataflow links with the $I D$ s 3 and 2. $f_{4}$ is scheduled on the dataflow links 3 and 8 . The integration specification model corresponding to this part is given by Figure 5. We illustrate only 


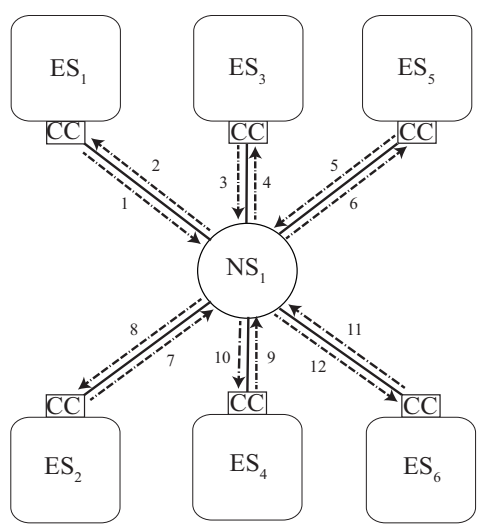

Fig. 4: Physical Architecture of the system

\begin{tabular}{|c|c|c|c|c|c|}
\hline \begin{tabular}{|c|} 
Integration \\
step \\
\end{tabular} & $\begin{array}{c}\text { Frame } \\
\text { Id } \\
\end{array}$ & \begin{tabular}{|l|} 
Frame \\
period
\end{tabular} & Availabilities & \begin{tabular}{|l|} 
Frame \\
length
\end{tabular} & $\begin{array}{c}\text { Virtual } \\
\text { Link }\end{array}$ \\
\hline \multirow{8}{*}{1} & $\overline{3}$ & $\overline{30}$ & {$[7,35,65,95]$} & 1 & 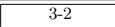 \\
\hline & 4 & 30 & {$[5,33,63,93]$} & 1 & $3-8$ \\
\hline & 40 & 60 & {$[21,21]$} & 2 & $3-2$ \\
\hline & 41 & 60 & {$[15,15]$} & 4 & $3-8$ \\
\hline & 42 & 60 & {$[17,17]$} & 1 & $1-4$ \\
\hline & 43 & 60 & {$[17,17]$} & 3 & $7-4$ \\
\hline & 1030 & 60 & {$[9,9]$} & 2 & $1-4$ \\
\hline & 1031 & 60 & {$[9,9]$} & 1 & $7-4$ \\
\hline \multirow{11}{*}{2} & 2 & 30 & {$[9,41,67,97]$} & 2 & 3-8 \\
\hline & 10 & 60 & {$[51,51]$} & 3 & $3-2$ \\
\hline & 11 & 60 & {$[31,31]$} & 7 & $3-8$ \\
\hline & 12 & 60 & {$[27,27]$} & 3 & $3-8$ \\
\hline & 13 & 30 & {$[25,61,75,107]$} & 4 & $7-2$ \\
\hline & 14 & 60 & {$[71,71]$} & 1 & $1-8$ \\
\hline & 15 & 60 & {$[89,89]$} & 2 & $7-4$ \\
\hline & 1000 & 30 & {$[17,53,89,105]$} & 1 & $1-4$ \\
\hline & 1001 & 30 & {$[19,55,69,101]$} & 2 & $7-4$ \\
\hline & 1002 & 60 & {$[63,63]$} & 1 & $1-4$ \\
\hline & 1003 & 60 & {$[7,7]$} & 1 & $7-4$ \\
\hline \multirow{7}{*}{3} & 0 & 60 & {$[35,95]$} & 1 & $9-4$ \\
\hline & 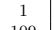 & 30 & {$[0,30,60,90]$} & 1 & $3-10$ \\
\hline & 109 & 60 & {$[22,82]$} & 11 & $11-\{2,4,6,8$, \\
\hline & 110 & 60 & {$[14,74]$} & 5 & $-\{2,4,6,8$, \\
\hline & 111 & 60 & {$[7,67]$} & 2 & $11-\{2,4,6,8$, \\
\hline & 140 & 60 & {$[61,81]$} & 4 & $3-6$ \\
\hline & & & & & \\
\hline
\end{tabular}

Table 1: Temporal characteristics of integrated frames

the schedules $s 1$ and $s 2$ of frames $f 3$ and $f 4$ on the dataflow link $l 3$. As shown

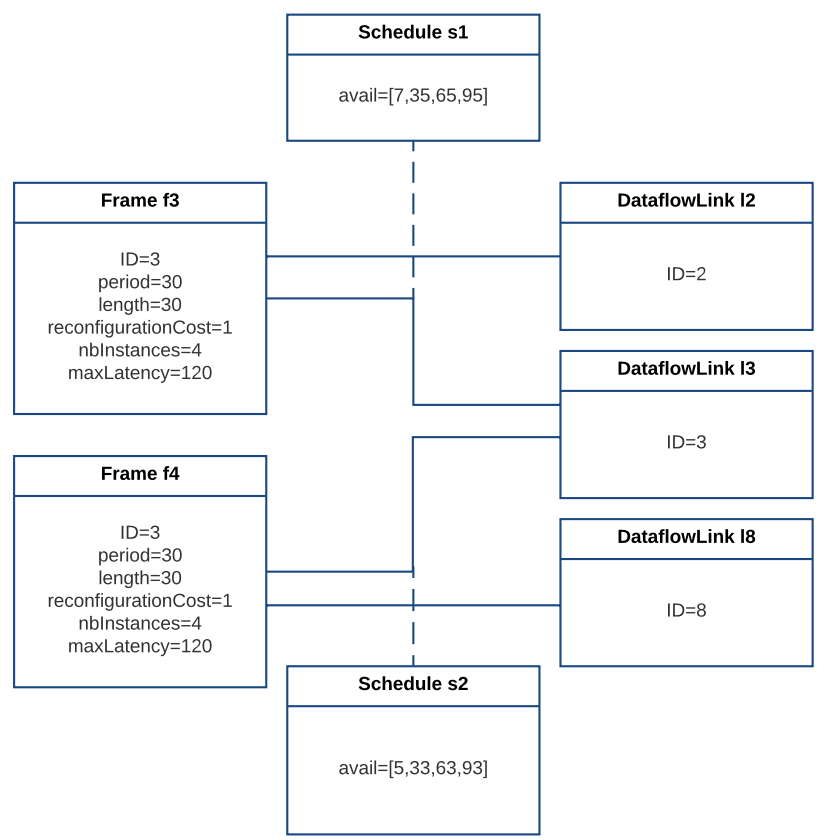

Fig. 5: Integration Specification Model of Example 1 
by Figure 6, two instances fio1 and fio2 of the class FrameInstanceOffsets are defined in our output CP model to represent the schedules $s 1$ and $s 2$. As the number of instances of $f_{3}$ considered in the integration specification model is equal to 4, the type attribute of the variable fio1 has a value of int[4]. The corresponding MiniZinc Code is given by Figure 7. We note that we limit our offsets to the interval [1..120] to have an enough large finite domain that represents the different possible values. Now that we have illustrated the different variables of

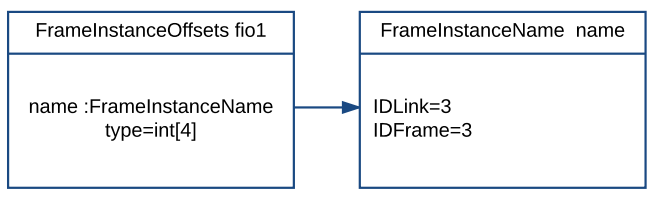

Offsets variable of frame $\mathrm{f} 3$ on dataflow link with ID 3

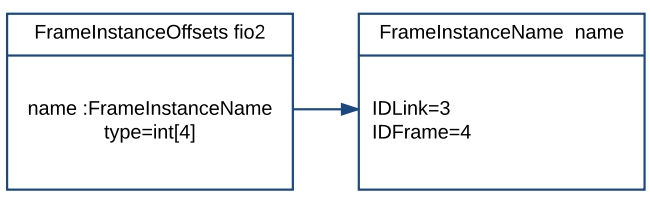

Offsets variable of frame $f 4$ on dataflow link with ID 3

Fig. 6: CP Variables considered in Example 1

$$
\begin{aligned}
& \text { array }[1 . .4] \text { of var } 0.120: \text { Link30ffset3; } \\
& \text { array }[1.4]
\end{aligned}
$$

Fig. 7: Declaration of the variables of Example 1 in MiniZinc

the CP Integration Model, we present the contention-free constraint of Example 1. This constraint is defined by the instance $c 1$ of the metaclass Contention FreeConstraint as illustrated by Figure 8 . The instance $c 1$ contains the information required to generate the corresponding MiniZinc code as illustrated by Figure 9

\subsection{Example 2}

The frame $f_{3}$ is transmitted on the virtual link $v l_{3}$ which is composed by the datapath $3-8$. The transmission of $f_{3}$ on its associated datapath induces the definition of a Path-Dependent Constraint that has the structure presented by Figure 10. In addition to the attributes switchDelay and length, to define this 


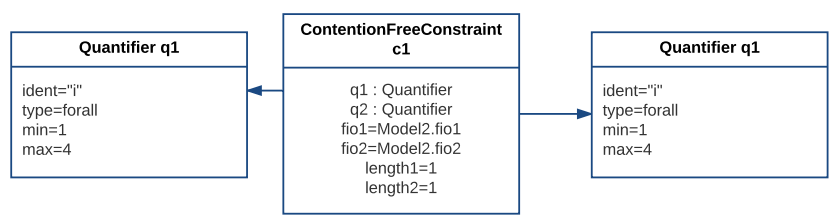

Fig. 8: Contention-Free Constraint considered in Example 1

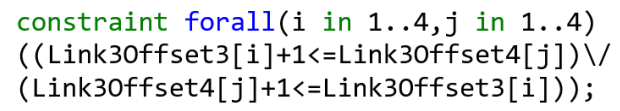

Fig. 9: Example 1:Contention-Free Constraint Code in MiniZinc

constraint, we consider from Example 1 the instance fio 1 to characterize the offsets of $f_{3}$ on the dataflow link 2. We consider also the instance fio3 that defines the offsets associated to the transmission of $f_{3}$ on the dataflow link 2 . The corresponding MiniZinc code corresponding to this constraint is given by Figure 11

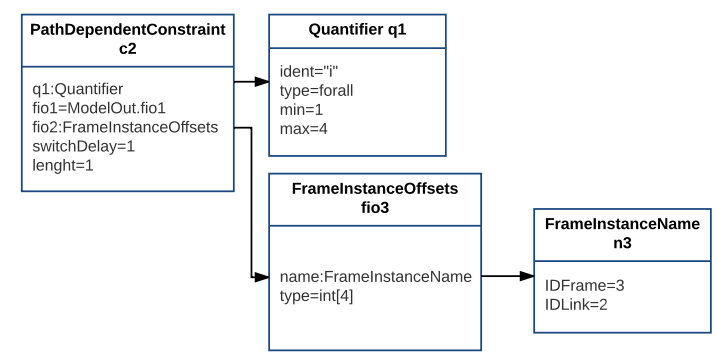

Fig. 10: CP Integration Model of Example 2

constraint forall ( $i$ in $1 . .4$ )

(Link30ffset3[i] $+1+1<=$ Link20ffset3[i]);

Fig. 11: Example 2: Path-Dependent Constraint Code in MiniZinc 


\section{Related Works}

The constraint programming approach to the scheduling problems in avionic systems is now a very active and popular research field. Several formal definitions and frameworks have been proposed for reasoning about the problem of scheduling in IMA architecture (e.g. [11, 10]), the problem of scheduling in TimeTriggered Networks (e.g $[14,15,12,7])$ and the cost optimization problems for evolving avionic systems (e.g. [9]). The most related work to ours is the recent paper of Lauer et al. [9] and the one of Steiner [14]. In [9], the authors address the problem of an iterative integration in an IMA Architecture. Its objective is to find an optimal scheduling configuration that minimizes the cost of the integration. However, it does only consider the integration of IMA partitions and the proposed iterative approach handles only the scheduling of system model that evolves by adding a single partition at each iteration. The work in [4] extends the work in [9] to consider a SMT-based approach that handles not only the integration of IMA partitions but also TTEthernet flows.

The combination of model-driven software engineering approach and constraints programming approach has been the focus of some other research works including [8] and [5]. In [8], the authors propose a formalization of constraint programming solving tasks in a model-driven process chain. In [5], the authors discuss the need for a visual high level modeling language and the quality of metamodeling techniques to implement the transformations. In particular, they present a platform called s-COMMA, which efficiently implements the chain from modeling to solving constraint problems.

\section{Conclusion}

In this paper, we have proposed a model-driven engineering approach to support the automatic synthesis of programs that resolve the integration of TT flows of TTEthernet. The proposed approach relies on the definition of two meta-models. The first one specifies an integration problem on TTEthernet networks. The second one describes the structure of the corresponding CP program. Further to the two meta-models, this approach is based also on the definition of transformation processes that automatizes the generation of the $\mathrm{CP}$ model to a given integration problem. The resulted CP model is transformed to a CP code which is resolved by a CP solver to find the new optimal configuration of the network. As Future Work, we plan to extend our approach by considering the schedule of IMA partitions. We expect no difficulties to extend the two meta-models to consider the integration of IMA partitions and their associated constraints. We will define also the necessary transformation process to automatize the synthesis of IMA constraints.

Acknowledgment This work was supported by the Natural Sciences and Engineering Research Council of Canada (NSERC). 


\section{References}

[1] Integrated modular avionics (ima). AERONAUTICAL RADIO, INC., ARINC 653, 2006.

[2] ARINC 664, part 7: Avionics full duplex switched ethernet (afdx). AERONAUTICAL RADIO, INC., 2009.

[3] AS6802: Time-triggered ethernet (ttethernet). SAE Aerospace, 2011.

[4] Sofiene Beji, Sardaouna Hamadou, Abdelouahed Gherbi, and John Mullins. Smtbased cost optimization approach for the integration of avionic functions in ima and ttethernet architectures. In Proceedings of the 2014 IEEE/ACM 18th International Symposium on Distributed Simulation and Real Time Applications, pages 165-174. IEEE Computer Society, 2014.

[5] Raphaël Chenouard, Laurent Granvilliers, and Ricardo Soto. Model-driven constraint programming. In Proceedings of the 10th international ACM SIGPLAN conference on Principles and practice of declarative programming, pages 236-246. ACM, 2008.

[6] Optimisation Research Group. Minizinc 2.0 . http://www.minizinc.org/ide/index.html, 022016.

[7] Jia Huang, Jan Olaf Blech, Andreas Raabe, Christian Buckl, and Alois Knoll. Static scheduling of a time-triggered network-on-chip based on smt solving. In Proceedings of the Conference on Design, Automation and Test in Europe, pages 509-514. EDA Consortium, 2012.

[8] Mathias Kleiner, Marcos Didonet Del Fabro, and Patrick Albert. Model search: Formalizing and automating constraint solving in mde platforms. In European Conference on Modelling Foundations and Applications, pages 173-188. Springer, 2010.

[9] Michaël Lauer, John Mullins, and Moez Yeddes. Cost optimization strategy for iterative integration of multi-critical functions in ima and ttethernet architecture. In Computer Software and Applications Conference Workshops (COMPSACW), 2013 IEEE 37th Annual, pages 139-144. IEEE, 2013.

[10] Y-H Lee, Daeyoung Kim, Mohamed Younis, Jeff Zhou, and James McElroy. Resource scheduling in dependable integrated modular avionics. In Dependable Systems and Networks, 2000. DSN 2000. Proceedings International Conference on, pages 14-23. IEEE, 2000.

[11] Y-H Lee, Daeyoung Kim, Mohammed Younis, and Jeff Zhou. Scheduling tool and algorithm for integrated modular avionics systems. In Digital Avionics Systems Conference, 2000. Proceedings. DASC. The 19th, volume 1, pages 1C2-1. IEEE, 2000.

[12] Rupak Majumdar, Indranil Saha, and Majid Zamani. Performance-aware scheduler synthesis for control systems. In Proceedings of the ninth ACM international conference on Embedded software, pages 299-308. ACM, 2011.

[13] Francesca Rossi, Peter Van Beek, and Toby Walsh. Handbook of constraint programming. Elsevier, 2006.

[14] Wilfried Steiner. An evaluation of smt-based schedule synthesis for time-triggered multi-hop networks. In Real-Time Systems Symposium (RTSS), 2010 IEEE 31st, pages 375-384. IEEE, 2010.

[15] Domitian Tamas-Selicean, Paul Pop, and Wilfried Steiner. Synthesis of communication schedules for ttethernet-based mixed-criticality systems. In Proceedings of the eighth IEEE/ACM/IFIP international conference on Hardware/software codesign and system synthesis, pages 473-482. ACM, 2012. 